\title{
TURBULENT FLOW BETWEEN CONCENTRIC ROTATING CYLINDERS
}

\section{S. Mohamed}

Meckanical Power Engincering Department, Facuity of Engineering, Massoura Udiversity.

$$
\begin{aligned}
& \text { الانسياب المضطرب بين أسطو اتتين دوارتين متحدنى العركز. }
\end{aligned}
$$

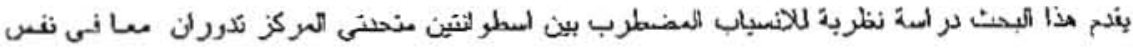

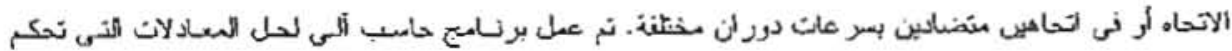

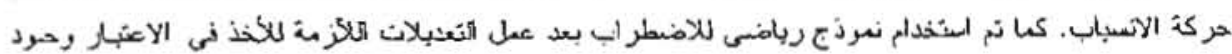

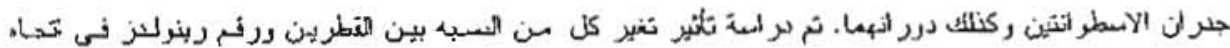

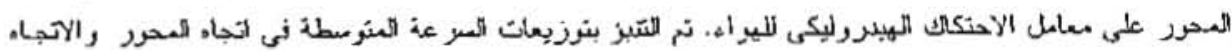

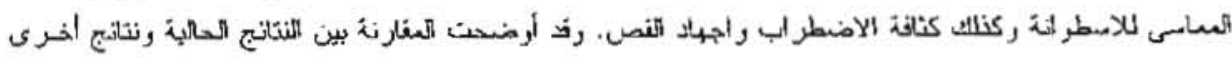

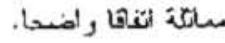

\begin{abstract}
:
The turbulent flow betweev concentric cylinders both rotating in the same or opposite directions is investigated theoreticaily. A finite difference scheme is developed 10 solve the boundary layer equations coverning the flow. A two-equation model of turbulence was employed in this study. Modifications have been wade to the model to take into account the effect of rotation of the cylinders and the existing of the wall. The effect of changing the radius ratio, axial Reynolds number and angular velocity ratio on the hydraulic friction head loss of air is studied. Distributions for axial mean velocity, tangential mean velocity, turbulence intensity and sliear stress in botb the entrance region and fully developed rcgion are predicted, aiso. Comparisons of the present numerical results wilb other investigators results show good agreemen.
\end{abstract}

\section{I.INTRODUCTION:}

Turbulent flow between concentric cylinders (one of them rotating) occurs in various rotating machinery such as joumal bearing, axial flow between the stationary and rotating parts of turbo-nachines and the flow in the rising line of deep well pumps. One of the important examples is the cooling system of gas turbine rotor, where some portion of the cooling air can be supplied to the rotor atea through an annuiar duct, fonned by two coaxial cyliuders, with the inner cylinder rotating. Flow in such system can be characterized by very high Reynoids oumber $\left(R_{e} \approx 10^{6}\right)$. Most of papers dealing with this type of flow analyze the problem in the range of low or moderate $R_{e}$, in an effort to explain the nature of Tayior vortices and to quantify their complex effects. There are very few data, botb experimental and theoretical, from the ravg of high $R_{e}$.

The papers of Yamada $[1,2]$ are dealing with high rotational $R_{e}$ and axial throughtlow. Kaye and Elgar [3] used the axial Reynolds number $\left(R_{e}\right)$ and Taylor number as criteria, characterizing the flow, to distinguish four distinct flow regimes. These rcgimes are laminar 
flow, laminar flow with Taylor vorices, turbulent flow and turbulent flow with Taylor vortices. The boundaries of the regimes tvere conveniently represented by a plot of the axiat Reynoids number versus Taylor number. The experiments of Kaye and Elgar [3] were extended to higher Reynolds and Tayior nuınbers by Kostcrin, Koshmanow and Finatyew, and are quoted in [4].

Mansour [5] investigated theoretically the hydraulic resistance in an annulus of concentric rotating cylinders. The axial and tangential velocity distributious were introduced. He assumed that the annulus is long enough and a uniform purely developed laminar flow is considered. He found that rotation affects the friction coefficient as well as the axial and tangential velocity distributions. He concluded that the friction coefficient for an annulus of rotating walis is higher than that for stationary walls. Also, the friction coefficient ratio increases with the increase of bolls speed ratio $(\omega / \mathrm{u})$ and the relative angular velocity ratio $\left(\omega_{1} / \omega_{2}\right)$.

Boubnov et al [6] investigated the stability conditions $c$ f the flow between two concentric cylinders with the inner one rotating. They cmployed a fluid with axial, stable linear density stratification. They used a gap of radius ratio 0.275 . Experiments show dat stratification bas stabilizing effect on the flow with the critical Reynolds oumber depending on the buoyancy frequency of the fluid. The sclccted vertical wave length at onset of instability is reduced by stratification effect and is for the geometry considercd only about half the gap width. They devided the flow regimes into three regimes depending on both Reynolds number and Froud number.

Although the stability of supercritical circular couctte flow has been studied extensively, results for the velocity field of the flow are limited. Recently, the azimuthal velocity profiles for the Taylor vortex, wavy vortex and turbulent Taylor vortex flow in the annuius between a rotating inner cylinder and a fixcd outer cylinder with fixed end conditions were mcasured using laser Doppler velocimetry by Wereley and Lueptow [7]. They measured the azimutbal velocity at several points distributed at both radial and axial directions for several Reymolds number. Increasing Reynolds number indicated that: the magnitude of the ratial gradient of azimuthal velocity near both cylinders increases and the radial outflow region between pairs of vortices becomes increasingly jet-like.

The present investigation is concemed with the theoretical study of the development of turbulent flow in concentric annulus at different conditions such as rotating the inner and outer cylinders in the same or opposite directions at different velocity ratios. Steady axial flow is assumed to exist under a constant axial pressure gradient. The aim of this study is to develop a finite difference scheme using the available turbulence modeis to predict the now patterns and its effect on the hydraulic loss coefficient.

\section{GOVERNING EQUATIONS:}

Assuming that the fluid las constant plysical properties and the flow is steady axisymmetric with the absence of body forces. The equations governing the fluid motion of an annulus with rotating cylinders and axial throughflow, in cylindrical polar coordinates, are:

$r$-monemum equation:

$$
V_{,} \frac{\partial V_{r}}{\partial r}-\frac{V_{n}^{2}}{r}+V_{,} \frac{\partial V_{r}}{\partial z}=-\frac{I}{\rho} \frac{\partial \rho}{\partial r}+v\left[\frac{1}{r} \frac{\partial}{\partial r}\left(r \frac{\partial V_{r}}{\partial r}\right)-\frac{V_{r}}{r^{2}}+\frac{\partial^{2} V_{r}}{\partial z^{2}}\right]
$$


$\theta$-momentum equation:

$$
V, \frac{\partial V_{\theta}}{\partial r}+\frac{V_{r} V_{0}}{r}+V, \frac{\partial V_{0}}{\partial z}=v\left[\frac{l}{r} \frac{\partial}{\partial r}\left(r \frac{\partial V_{0}}{\partial r}\right)-\frac{V_{0}}{r^{2}}+\frac{\partial^{2} V_{0}}{\partial z^{2}}\right]
$$

$z$-momentum equation:

$$
V_{1} \frac{\partial V_{t}}{\partial r}+V_{t} \frac{\partial V_{1}}{\partial z}=-\frac{l}{\rho} \frac{\partial P}{\partial z}+v\left[\frac{1}{r} \frac{\partial}{\partial r}\left(r \frac{\partial V_{t}}{\partial r}\right)+\frac{\partial^{2} V_{t}}{\partial z^{2}}\right]
$$

Contimuity equation:

$$
\frac{\partial\left(r V_{s}\right)}{\partial z}+\frac{\partial\left(r V_{r}\right)}{\partial r}=0
$$

Scalar property $\phi$ :

The transport equation of the scaiar quastities: turbulent kinetic energy $\dot{k}$, its dissipation rate $\varepsilon$ and the Reynolds stresses in a general from is:

$$
\rho \frac{\partial}{\partial z}\left(r V_{*}\right)+\rho \frac{\partial}{\partial r}\left(r V_{t \rightarrow}\right)=-\frac{\partial}{\partial r}\left(r J_{t \rightarrow}\right)+P_{\theta}-D_{\phi}
$$

where $\phi$ is the required scalar quantity. $J_{+}$is the fux of the scalar property $\phi, P_{\phi}$ is the generation rate of $\phi$ and $D_{0}$ is the dissipation rate of $\phi$. The velocity components $\left(V_{z}, V_{r}\right.$ and $V_{\theta}$ ) and the scalar property $\phi$ represenz tiune average values.

Applying the well known Prandtl boundary layer assumptions and check the order of magnitude of each term of the above equations [8], equations (1), (2) and (3), which represent the momentum equations for the flow in the entry region, may be reduced to the following simplified boundary layer equations:

$$
\begin{gathered}
\rho \frac{V_{0}^{2}}{r}=\frac{\partial P}{\partial r} \\
V_{r} \frac{\partial V_{0}}{\partial r}+V_{1} \frac{\partial V_{\theta}}{\partial z}=\frac{v}{r} \frac{\partial}{\partial r}\left(r \frac{\partial V_{0}}{\partial r}\right) \\
V_{r} \frac{\partial V_{1}}{\partial r}+V_{r} \frac{\partial V_{t}}{\partial z}=-\frac{1}{\rho} \frac{\partial P}{\partial z}+v\left[\frac{1}{r} \frac{\partial}{\partial r}\left(r \frac{\partial V_{1}}{\partial r}\right)+\frac{\partial^{2} V_{1}}{\partial z^{2}}\right]
\end{gathered}
$$

The fully developed flow, which occurs if the annulus is sufficiently long, provides an analytical clieck on the finite difference solution to be obtained. Aftcr passing the initial section, all derivatives in the axial direction become zero, except the constant pressurc gradient $(\partial / \partial z)=0$. Additionally, there are no variation in the tangential direction $(\partial / \partial \theta)=0$, because of the axial symnetry of the system. With these simplifications and the assumptions of negligible dissipation rate and no body forces, except the centrifugal force, the equations of conservation for an incompressible turbulent flow will take the form: 
$A^{0} .=(D+(\mathrm{C} / 2))^{D}, \quad A_{j}^{D}=A_{S}^{U}+A_{j}^{D}+A^{D}$.

$B_{1}=\int_{X^{0}}^{X_{\eta}} \prod_{\eta} r \delta S_{+} d z d \eta=\overline{s_{*}}$ Volum. $\quad$ (Volume $\left.=r \delta \nabla z \nabla \eta\right)$

where $\overrightarrow{s_{\phi}}=$ Average source $(\sin k)$ vaiue over the control voiume.

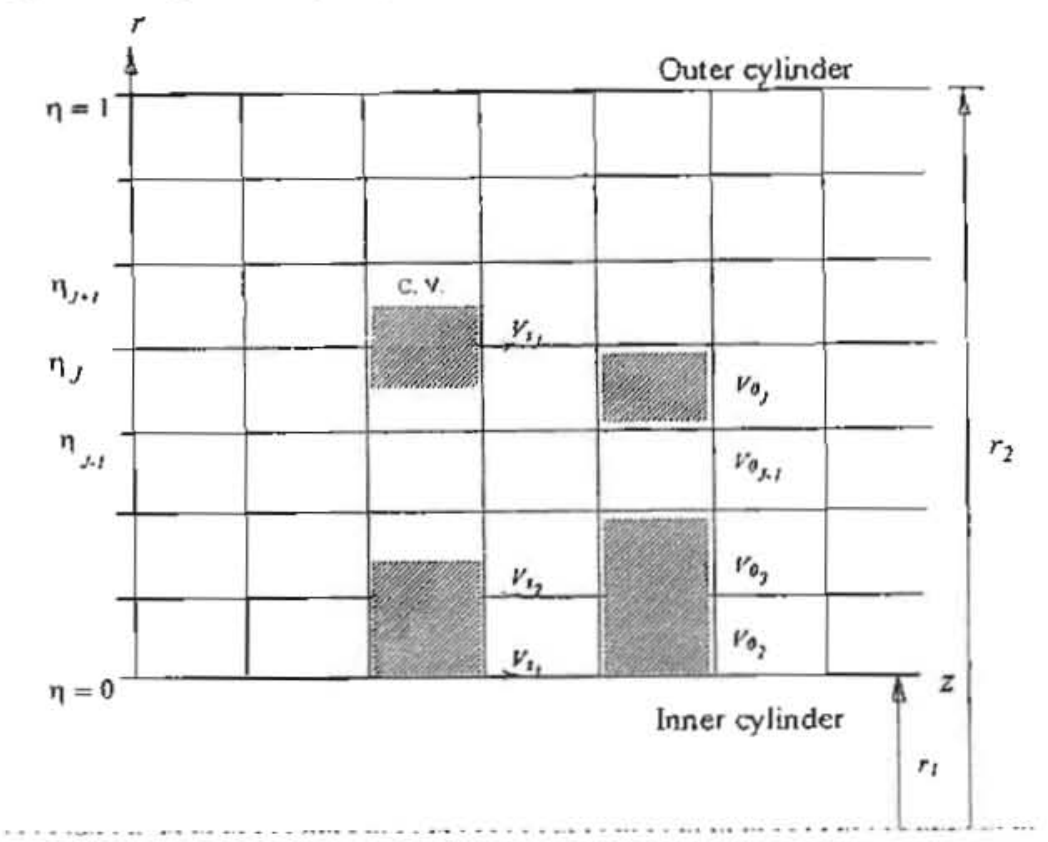

Axis of symmetry

Figure (i) Mesh network and control volumes for finite difference representation

Integrating the continuity equation over the $V_{\mathrm{g}}$-control volume gives:

$$
\left[\left(\rho r \delta V_{,} \nabla \eta\right)^{0}-\left(\rho r \delta V_{*} \nabla \eta\right)^{\nu},\right]+\left[\rho r\left(V_{\theta}-G V_{0}\right) \nabla z\right] \cdot-\left[\rho r\left(V_{\theta}-G V_{0}\right) \nabla z\right]=0
$$

$\left(V_{\theta}\right)_{+}$equals to $\left(V_{\theta}\right)_{l+1}$ and $V$. equals to $\left(V_{\theta}\right)_{J \cdot 1}$.

The equation of $V_{z}$ can be obtained by replacing $\phi$ by $V_{z}$ in equation (18), as follows:

$$
A_{j}^{D}\left(V_{2}\right)_{j}^{D}=A_{j}^{U}\left(V_{2}\right)_{j}^{U}+A_{j}^{D}\left(V_{1}\right)_{j, 1}^{D}+A_{j}^{D} \cdot\left(V_{1}\right)_{j-1}^{D}+B_{j}
$$

where the constants $A$ are coefficients of iniegration, superscript $U$ denotes upstream conditions while $D$ denotes dowastream conditions and $B_{j}$ is:

$$
B_{1}=-(r \delta)_{j}^{n} \nabla z \nabla \eta(\overline{d P} / d z)
$$

In confined flow $(\overline{d P} / d z)$ is not known. An estimated pressure field $(\overline{d P} / d z)^{*}$ is used with the transport equation of $V_{z}$-momentum to obtain a preliminary $V_{z}$-velocity distribution, say $\left(\left(V_{1}\right)^{D},\right)^{*}$. This velocity field satisfics the momentum equation but it does not satisfy the 
continuity equation. The pressure and velociry are then corrected in the following wa [13]: To estimate the mass-flux imbalance at each node, a veiocity cortection $\left((V,)_{j}{ }^{D}\right)$ was sdded to $\left(\left(V_{,}\right)^{0},\right)^{*}$ in order to satisfy the continuity equation. All approximate relation between $\left((V,)^{n},\right)^{\prime}$ and the cotresponding pressure correction $(\overline{d P} / d z)$ can be obtained Grom u-momentum cquation. Solving both equations will lcad to the pressure and velocity corrections, $\left(\left(V_{1}\right)^{n},\right)^{\prime}$ and $(\overline{d P} / d z)^{\prime}$ respectively. Better approximations for the pressure gradient and the velocity field can be obtained as:

$$
\begin{aligned}
& \overline{d P} / d z=(\overline{d P} / d z)^{\circ}+(\overline{d P} / d z)^{\prime} \\
& \left(V_{1}\right)_{,}^{D}=\left(\left(V_{l}\right)_{j}^{D}\right)^{\cdot}+\left(\left(V_{2}\right)_{j}^{D}\right)^{\prime}
\end{aligned}
$$

The new velocity field satisfied the continuity equation exactly. But, the substitution with the new pressure gradient in the $V_{z}$-momentum equation will lead to a velocity field which does not satisfy continuity equaticii exactly. An iterative procedure is needed before marching downstream to the next cross-stream plane until the difference is less than $0.3 \%$.

By the same manner, equation of $V_{\theta}$ is:

$$
A_{j y}^{D}\left(V_{0}\right)_{j}^{D}=A_{l y}^{u}\left(V_{0}\right)_{j}^{u}+A_{j, V}^{D}\left(V_{0}\right)_{j, y}^{D}+A_{j, y}^{D}\left(V_{0}\right)_{j, 1}^{D}+B_{y,}
$$

where

$$
B_{y,}=-(r \delta)_{j}^{n} \nabla_{z} \nabla \eta\left(p_{j-1}^{D}-p_{s}^{D}\right)
$$

$A$-coefficients and $B$-coefficient have the subscript $V_{\theta}$ because they are obtained from integration of equation (5) over the $V_{\theta}$-control volume. The pressure fluctuation $P_{j}^{D}$ is unknown. The same procedure in finding the pressure gradient and $V_{z}$-field is followed also to find the pressure fluctuation and the $V_{\theta}$-Geld from $V_{\theta}$-momentum equation. It gives:

$$
\begin{aligned}
P_{j}^{D} & =\left(P_{j}^{D}\right)^{*}+\left(P_{J}^{D}\right)^{\prime} \\
\left(V_{0}\right)_{j}^{D} & =\left(\left(V_{0}\right)_{j}^{D}\right)^{*}+\left(\left(V_{0}\right)_{j}^{D}\right)^{\prime}
\end{aligned}
$$

\subsection{Initial and Boundary Conditions:}

Uniform axial velocity profile is assumed at the inlet of the annulus and equais to the mean velocity. The radial and tangential veiocity profiles are assumed to be zero. The initial distribution of turbulent kinetic energy $k$ and its dissipation rate $\varepsilon$ are assumed be to unjorm and equal to that values given by Launder and Spalding [14] for turbulent pipe flows. All velocity components along the inner and outer radiuses are assumed to be zero, expect for the tangential mean velocity at the wall of the inner and outer cylinders. For an annulus having an inner cylinder of radius $r_{\text {, }}$ and rotates with angular velocity $\omega_{1}$ while its outer cyliuder is of radius $r_{2}$ and rotates, also, with angular velocity $\omega_{2}$, the flow is subjected to the following boundary conditions:

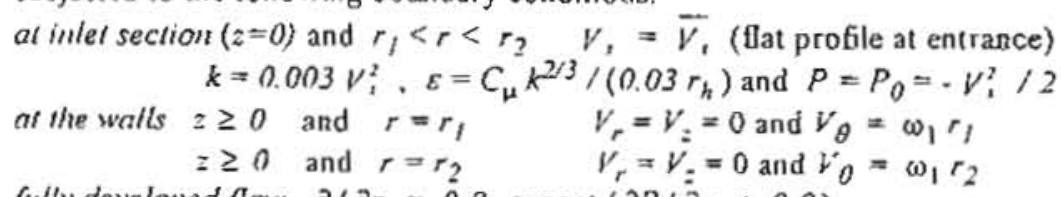

fully developed fow $\partial / \partial \mathrm{z}=0.0$ except $(\partial P / \partial \mathrm{z} \neq 0 . \Omega)$ 


\subsection{Near Wall Treatment:}

The $k-\varepsilon$ is valid only in bigh Reynolds number flows far from the viscous flow near the wall. Therefore, special treatment is needed to take into account the effect of fludd viscosity on turbulence, particularly, wall shear stress $\tau_{\mathrm{w}}$, the production of turbulence energy $k$ and its dissipation $\varepsilon$ near the wall. The procedure is based on the analytical solutions of the thin Coutte-llow type region which extends from $r^{2} \approx 30$ to the wall, where $\left(r^{+}=r \tau_{w} / v\right)$. This procedure is biased on the assumption that the Log-law:

$$
\frac{V_{\theta}}{\left(V_{\theta}\right)_{r}}=\frac{I}{\mathrm{x}} \ln \left(E_{r^{*}}\right)
$$

is valid over the uniform shear stress region $\left(0<r^{+}<r_{p}\right)$ where

$\left(V_{\theta}\right)_{s}=\sqrt{\tau_{\omega} / p}, \kappa=0.42$ (Von Karman constant) and $E=9.0$ for sroootb walls.

Using the simplification [11]

$$
\tau_{p}\left(=\tau_{w}\right)=p C_{\mu}^{i / 2} k_{p}
$$

to caiculate $\left(V_{B}\right)_{P}$ the wall sinear stress $\tau_{w}$ can be obtained as:

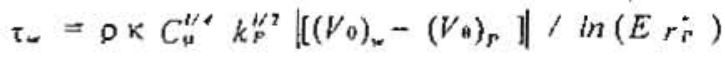

$$
\begin{aligned}
& r_{p}=\rho r_{p} C_{\mu}^{1 / \alpha} k_{p}^{1 / 2} / \mu
\end{aligned}
$$

subscripts $p$ and $w$ denotes the node $p$ and the wall conditions, respectively. In cquation (27), the quantity $\left.\left[\left(V_{\theta}\right)_{w}-V_{\theta}\right)_{P}\right]$ is employed to account for the rotation of walls.

The production of turbulence near the wall can be obtained from transport equation $k$. It was modified in order to take into account the effect of wall sbear stress $\tau_{w}$ From equation (16) the term, $-\overline{v_{s}} v_{r}\left(\partial V_{r} / \partial r\right)$, is the dominated part of $P_{k}$ and is denoted as $P_{k}^{\prime}$.

$$
P_{i}=-\overline{v_{r} v_{r}} \frac{\partial V_{2}}{\partial r}=\frac{\tau_{n}}{\rho} \frac{\partial V_{2}}{\partial r}=\frac{\tau_{w}}{\rho} \frac{\left(V_{0}\right)_{r}}{k r}
$$

therefore,

$$
P_{i}^{\prime}=\tau_{w}^{3 / 2} / \rho^{3 / 2} \times r \text {. }
$$

The dissipation rate near the wall, $\varepsilon$, was obtained from the assumption that the length scale of turbulence is $L=k^{3 / 2} / \varepsilon$ which is reiated to the mixing length as $L=C_{\mu}^{-3 / 2} \mathrm{~K} r$, . thus,

$$
\dot{\varepsilon}=C_{\mu}^{3 / 4} k_{p}^{3 / 2} / \mathrm{k} r
$$

\section{RESULTS AND DISCUSSION:}

The axial air low in four gaps, at radius ratio of $0.333,0.5,0.6$ and 0.75 , rotating at wide range of angular velocity ratios is used in this study. Positive and negative angular velocity ratios as well as inner cylinder rotating only and outer cylinder rotating only were investigated to give a range of flow Reynolds number $6.62 \times 10^{5} \leq R_{e} \leq 16 \times 10^{6}$ and rotational Reynolds numbers $2.43 \times 10^{3} \leq R_{0} \leq 48 \times 10^{4}$. The effect of these parameters on the flow 
pattern and friction head loss was studied. In all numerical computations a non-uniform grid in the radial direction is employed depending on the axial velocity gradient. Tweuty nodes in the radial direction were used to give grid-independent solution for this flow. Five itcration were found to give a converging solution.

\subsection{Lass Coefficient in Rotating Concentric Pipes:}

In all the test runs axial Reynolds number $R_{e}$ was higher than $10^{5}$, so the flow is turbulent when boch pipes are beld stationary. Therefore, for $R_{\theta}=0$, the friction head loss coefficient is equal that value calculated from 8lasius equation for smooth pipes $f=0.3164 R_{\text {, }}^{0.3 s}$.

When rotation begins, it is obvious that $\lambda=\left(\tau_{r 2}\right)_{W} /(1 / 2) V^{2}$, deviates gradually from $f$. Rotational hydraulic friction head loss $\lambda$ increases with increasing the aspect ratio $(\nu b)$ and reaches a constant vaiue at $1 / b=50$.

Figure (1) shows the variation of the friction coefficient ratic $\lambda f$ with radius ratio at different angular speed ratios $\alpha$ for aspect ratio $(U / b=S 0.0)$. The figure indicated that increasing the angular velocity ratio increases the friction coeffcient ratio $\left(R_{\theta} / R_{e}\right.$ increases). For constant angular velocity ratio and constant axial velocity $V_{z}=50(\mathrm{~m} / \mathrm{s})$, it is clear that $\lambda / f$ increases with increasing the radius ratio figure (1 a). For the case when outer cylinder is rotating only $\left(\omega_{1}=0\right)$, increasing the radjus ratio has an opposite effect on $\lambda f$ where it decreases with increasing the radius ratio as shown in figure $(1-b)$. This can be explained as, increasing the radius ratio decreases the tangential velocity gradient which in tum reduces the friction coefficient ratio. The figure shows that the friction coefficient ratio is greater than unity $(\lambda / f \geq 1)$.

The change of the friction coefficient ratio $N f$ with axial Reynolds number $R_{e}$ in a gap at radius ratio $=0.5$ for different angular velocity ratios is presented in figure (2). Figure $\{2-a)$ shows that for constant angular velocity ratio, $\lambda /$ increases with increasing $R_{e}\left(R_{\theta} / R_{e}\right.$ decreases as $R_{\theta}$ is constant). For outer cylinder rotating only $\left(\omega_{1}=0\right)$ shown in figure (2b), $\lambda f$ increases with increasing the angular velocity $\omega_{2}$ also, while it decreases with increasing the $R_{e}$ (or decreasing $R_{g} / R_{e}$ ).

The variation of the friction coefficient ratio with the rotational speed ratio... be explained by examining the velocity profiles in the gap of the rotating cylinders.

\section{2. Mean Velocity Distribution and Turbulence Intensity:}

The deveiopment of the flow parameters with the downstream distance for a gap having radius ratio 0.75 ; rotating at angular speed ratios $\alpha=2$, outer cylinder rotating only, inner cylinder rotating only and $\alpha=4$; are presented in figures (3 to 6) respectively. Comparison between the flow parameters for stationary (dashed lines) and rotating cylinders (solid lines) are presented. The dimensionless mean axial and tangential velocity profiles $\left[V_{t}\right.$ $\left\langle\left(V_{z}\right)_{\max }\right.$ and $\left.V_{\theta} /\left(V_{\theta}\right)_{\max }\right]$, turbulence intensity profiles $\sqrt{V_{r}^{j}} /\left(V_{z}\right)_{\max }$ and shear stress distribution $\overline{v_{r} v_{1} /\left(V_{t}\right)_{\max }^{2}}$ in the gap verses the dimensionless radial distance $\left(r_{-} r_{l}\right) /\left(r_{2}-r_{l}\right)$ are predicted for aspect ratio $/ / b=50$. Seven downstream stations $(z / L=0.04,0.12,0.3$, $0.45,0.6,0.75$ and 1.0 respectively) for a flow at axial Reynolds number $R_{e}=6.62 \times 10^{3}$ ivere chosen. $\eta_{1}$ dimensionless axiai mean velocity $V_{2} /\left(V_{2}\right)_{\max }$ was found to be self- 
similar after $z / L=50$. The velocity profiles $V_{z} /\left(V_{z}\right)_{\max }$ change along the annulus, as shown in the figures, for various values of $R_{f}$. The axiai mean velocity profiles remain flat up to distance $7 \pi,=0.30$. As $z / L$ increases, the velocity profiles deform gradually, due to a stabilizing effect of the centrifugat force of the swirliug flow. Lavan et ai [15] indicated in their theoretical svork that reverse flory is to be expected near the wall at the entrance region of the pipe, for large $R_{\theta}$. At $z / L=50$ and more downstream, the velocity profiles become approximately independent of the axial distance from the inlet. For a gap at constant radius ratio, the comparison between the mean axial velocity profiles at deferent angular velocity ratios indicated that the maximum velocity tends to move far from the axis of rotation as the angular velocity ratio increases winilc it is not affected by increasing axial Reynolds number $R_{e}$, as shown in figure (8-a). For constant angular velocity ratio, increasing the radius ratio tends to shift the maximum axial velocity towards the axis of rotation as sbown in figure (9-a), which agrees with Mansour [S] for laminar flow.

Figure (7) shows the response surface for the development of the dimensionless tangential mean velocity $V_{\theta} /\left(V_{\theta}\right)_{\max }$ for different angulai velocity ratios for a gap at radius ratio 0.6 and axial Reynolds number $R_{e}=6.62 \times 10^{5}$.

When both cylinders are rotating at opposite directions at $\omega_{1} / \omega_{2}=-2$ and axial Reynolds number $R_{e}=6.62 \times 10^{5}$, the Dow at every point is turbulent. Figure (3-b) shows that, the tangential mean velocity distribution near both cylinder walls is sharply inclined, whereas it is much less than that in the middle of the gap. As $R_{\theta}$ is increased, this trend is more. noticed. The turbulence intensity $\sqrt{v_{t}^{2}} /\left(V_{s}\right)_{\max }$ figure $(3-c)$, shows a peak near both cyliader walls. It was found that, this peak value increases as $R_{\theta}$ grows larger. Yamada [16] noticed that, once $R_{\theta}$ exceeds 5040 , the fuid is divided into two distirct regions of laminar and turbulent flows. This is the formation of a so-called spiral turbulence in which a laminar flow portion and turbulent Dow portion coexist like a double-screw pattern. He aiso noticed that, when both cylinders rotate in opposite directions, Taylor vortices and spiral turbulesce develop simultaneously or one after the other, depeading upon the rotational Reynolds number $R_{\theta}$, and they coexist in the gap.

When the outer cylinder is rotating at $R_{\theta}=7274$ wille the inner one is stationary $\left(\omega_{1}=0\right)$, the iurbuience intensity $\sqrt{\overline{v_{r}^{2}}} /\left(V_{2}\right)_{\max }$, sharply increases as shows in figure (4-c) and the velocity gradient (v) increases slightly, figure (4-b). Yamada [16], found the same phenomenon in bis experiment, and he noticed that the flow becomes spiral turbulence.

Figure (S) shows an axial flow at $R_{\mathrm{e}}=6.62 \times 10$ with the inner cylinder rotating at $R_{\theta}=2425$ while the outer cylinder is stationary $\left(\omega_{2}=0\right)$. The tangential mean velocity profice, figure (5-b). shows a flat portion, in the neddle, up to $\mathrm{z} / \mathrm{L}=0.6$. The velocity distribution shows self-similarity case after $/ / b=50$. The turbulence intensity $\sqrt{v_{z}^{2}} /\left(V_{z}\right)_{\max }$ figure (5-c), las a peak value near the inner cylinder and the axis of the fow tends to move towards the outer cylibder. This peak value decreases with increasing both the radius ratio and the axial Reynolds number $R_{\mathrm{e}}$. These results agree well with that obtained with El-shaarawi et al [17] for turbulent fiow in an annulus with inner cylinder rotation.

When the outer cylinder and the inner cylizder are rotating in the same direction $\left(\alpha=\omega_{1} / \omega_{2}=\right.$ $4)$, shown in figure $(6-b)$, the tangential mean velocity profile indicated two peak values near the rotating wails with bigh velocity gradient. The velocity gradient reduces as the tow goes downstream. The turbulence intensity, figure $(6-c)$, increases gradually from the outer to the inser cylinder and it has a steep change near the inner one. In the fully 
developed region, the flow is stabilized and Yamada [16] found that, the flow is lamiaar even at $R_{\theta}=5000$, because the laminar flow regime increases up to a high $R_{\theta}$ as the angular velocity ratio approaches unity.

\subsection{Shearing. Stress:}

The dimensionless sbear stress, $\overline{v_{t}} \bar{v}_{t} /\left(V_{x}\right)_{\text {mar }}^{2}$, jocreases as the flow goes down-stream as shown in figure (3-d). It decreases with increasing both the radius ratio and axial Reynolds number, figures ( 8 and 9 ). The shear stress was found to be reduced as the rotational Reynolds number increases. In the case of outer cylinder rotating while the inner cylinder is fixed, an opposite treod is obtained.

\section{CONCLUSIONS:}

Nonswirling turbulent axial flow between two concentric totating cylinders was studied oumerically. The flow pattern and the hydraulic resistance in the gap were investigated in the ranges of flow axial Reynolds number $6.62 \times 10^{5} \leq R_{e} \leq 5.16 \times 10^{6}$ and rotational Reynolds numbers $2.43 \times 10^{3} \leq R_{\theta} \leq 7.48 \times 10^{4}$. The flow parameters io both entrance and fully developed regions were predicted.

The results can be concluded in the following points:

1) For constant axial Reynolds number $R_{\mathrm{e}}$, the bydraulic friction coefficient, $\lambda / E$, in the initial region is function the rotational Reynolds number $R_{\theta}$ and the downstream distance $z / \mathrm{L}$ while after $z / \mathrm{L}>50$, it depends on the rotational Reyoolds number $R_{\theta}$, oniy.

2 ) In the fully developed region, the hydraulic friction coefficient increases with increasing $\left(R_{\theta} / R_{\mathrm{e}}\right)$. For the same the angular velocity ratio, it increases with increasing both $\left(R_{\theta}\right.$ $/ R_{\mathrm{e}}$ ) and radius ratio $r_{1} / r_{2}$. It is generally less than unity except for the case of the cylinders rotating in opposite directions.

3) In the case of outer cylinder rotating while the inner one is stationary; the friction coefficient ratio, $\lambda / \mathrm{f}$, increases with increasing the angular velocity of the outer cyinder $\omega_{2}$. For constant $\omega_{2}$, the swirling velocity given to the fow by cotating the cylioder tends to shit the flow gradually to laminar flow which in tura reduces the friction bead loss coefficient ratio. Reducing the radius ratio increase the rotating area which in turn allows the flow more to reach faminat flow case that decreases $\lambda \in \mathbb{E}$. The friction coefficient ratio, $\lambda / \mathfrak{f}$, is geuerally greater than unity.

4) The axial maximum mean velocity tends to shitt towards the axis of rotation as the radius ratio increase while there is no effect of increasing the axial Reynolds number $R_{\mathrm{e}}$ on the location of the axial maximurn velocity.

5) The turbulence intensity and shear stress reduce with increasing botb the axial Reynolds number and sadius ratio. The effect of increasing the rotational Reyoolds number is to reduce both turbulence intensity and shear stress while they increase with increasing $\omega_{2}$ in the case of stationary inner cylinder.

\section{NOMENCLATURE:}

b Gap width $=r_{2}-r_{1}$.

$C_{\varepsilon 1}, C_{\varepsilon 2} \quad$ Constants in the $k-\varepsilon$ model.

$C_{\varepsilon 3} \quad$ Constant for the additional dissipation term.

$C_{\mu} \quad$ Eddy viscosity coefficient.

$f$ Hydraulic friction coefficient for stationary cylinder. 
k Turbulent kinetic energy.

$L \quad$ Pipe length.

$P_{k} \quad$ Production term in the $k$ transport equation.

$R_{e} \quad$ Axial Reynolds number $=2 \mathrm{Ub} / \mathrm{v}$.

$R_{\theta} \quad$ Rotational Reynolds nurober $=\left|\omega_{2}-\omega_{1}\right|\left(r_{2}^{2}-r_{1}^{2}\right) / 20$.

$r \quad$ Axbitrary radius in the gap.

$r_{h} \quad$ Hydraulic radius $=2\left(r_{2}-r_{l}\right)=2 b$.

$r_{1}, r_{2} \quad$ Radius of inner and outer cylinder respectively.

$M=r_{1} / r_{2} \quad$ Radius ratio.

$r, \theta, z$

Cylindrical coordinate system.

$T_{k}, T_{\varepsilon}$

$V_{2}, V_{8}, V_{r}$

Diffusion term in $k$ transport equation and $\varepsilon$ transport equation respectively.

$v_{s}, v_{8}, v_{r}$ Axial, tangential and radial mean velocity respectively.

$\overrightarrow{v, v}, \quad$ Reynolds shear stress.

$\varepsilon$

$\omega$

$\rho$

$\mu$

$v$

$v_{t}$

$\sigma_{k, t}, \sigma_{\varepsilon, t}$

$\tau$

$\alpha$

$\lambda$.

Dissiparion rate of the turbulent kinetic energy $k$

A.ngular velocity.

Fluid density

Fluid dynamic viscosity.

Fluid kinematic viscosity.

Turbulent eddy viscosity.

Turbuleot Pratidt nusmber in $k$ and $\varepsilon$ transport equations.

Shear stress

Angular velocity ratio $=\omega_{i} / \omega_{2}$

Rotational bydraulic friction coefficient.

\section{REFERENCES:}

1-Yamada; Y., "Resistance of a Flow Througb an Annulus with an Inner Rotating Cyliader," Bulletin of J.S.M.E., Vol. S, No. 18, 1962, P. 302-310.

2-Yamada, Y., "Torque Resistance of a Flow Between Rotating Coaxial Cyliaders Having Axial Flow," Bulletin of J.S.M.E., Vol. 5, No. 20, 1962, PP. 634-642.

3-Kaye, J, and Elgar, E. C., "Modes of Adiabatic and Diabetic Fluid Flow in an Lner Rotating Cylinder," ASME Transactions, Vol. 80, 1958, PP 753-765

4-Beranek, K, and Streda, J., "On the Flow of Viscous Liquids Through Annular Clearance with the Rotating Inner Cylinder," Acta Technica CSAV, No. 6, 1979, PP $665-676$.

5-Mansour, H., "Laminar Flow Between Coaxial Rotating Cylinders," Mansoura Engineering Joumal (MEJ), Vol. 14, No. 2, Dec. 1989, PP M21- M4 I.

6-Boubnov, B. M., Gledzer, E. B., and Hopfinger, E. J., "Stratified Circular Couette Flow: Instability and Flow Regimes," Journal of Flud Mechanics, Vol. 292, Jun. 1995, PP 333.358 .

7-Wereley, S. T., aod Lueptow, R. M., "Azimuthal Velocity in Supercritical Circular Couette Flowv," Experiments of Fluids, Vol. 18, No. 1-2, Dec 1994, PP 1-9. 
8-Astill, K. N., "Modes of Adiabatic Flow in the Entrance Region of an Annuius with an Inner Rotating Cylizder," P/t.D. dissertation, Massachusetts Institute of Technology, 1961 .

9- Prandtl, L., "Bemerkungen zur Theorie der Frien Turbulenz", Lenz. ZAMM, Vol. 22, 1942, PP 241-243.

10- Kolmogorov, A. N., "Equations of Turbulent Motion of an Jocompressible Fluid", Itv. Ak nauk SSR, Serra Fizicheska, VI 1942, No. 112, 1942, PP 56-58.

11-Patanker, S. V. and Spalding, D. B., "A Calculation Procedure for Heat, Mass and Momentum Transfer in Three-Dimensional Parabolic Flows, "International Journal of Heat and Mass Transfer, Vol. 15, 1972, PP 1787-1806.

12-Jones, W. P., and Launder, B. E., "The Prediction of Laminarisation with a TwoEquation Model of Tubulence," Intemational Journal of Heat and Mass Tramsfer, Vol. 15, 1972, PP 301 .

13-Carretto, L. S., Gosman, A. D., Patanker, S. V. and Spalding, D. B., "Two Calculation Procedures for Steady. Three-Dimensional Flows with Recirculation," Proceeding 3rd International Conference on Numerical Methods in Fluid Dynamics, Springer-Verlag, New York, 1972, PP 60-68.

14-Launder, B. E., and Spalding, D .B., "The Numerical Computation of Turbuient Flows," Computer Methods in Applied Mechanics and Engineerng, Vol. 3, 1974, PP 269-289.

15-Lavan, Z., Nielsen, H., and Fejer, A. A., "Separation and Flow Reversal in Swirling Flows in Circular Ducts," Physics of Flunds, Vol 12, No. 9, 1969, PP 1747-1757.

16-Yamada, Y., and Imao, S., "Flow of a Fiuid Contained Between Concentric Cylinders Both Rotating," Bulletin of J.S.M.E., Vol 29, No. 252, 1986, PP. 1691-1697.

17-El-Shaarawi, M., and Sarhan, S., "Developing Lamizar Free Convection in an Opea Ended Vertical Annulus with a Rotating [nner Cylinder," ASME Journal ; Fluid Engineering, Vol. 103, Aug. 1981, PP 552-558

18-Morcos. S. M., Abou-EUail, M. M., Abou-Arab, T..W., "Corobined Forced and Free Turbulent Heat Transfer in a Verical Annulus with Rotating Inner Cylinder," $3 \underline{r d}$ International Conference on Methods in Thermal Problems, Seattle, Washington, August, 1983, PP 762-777. 


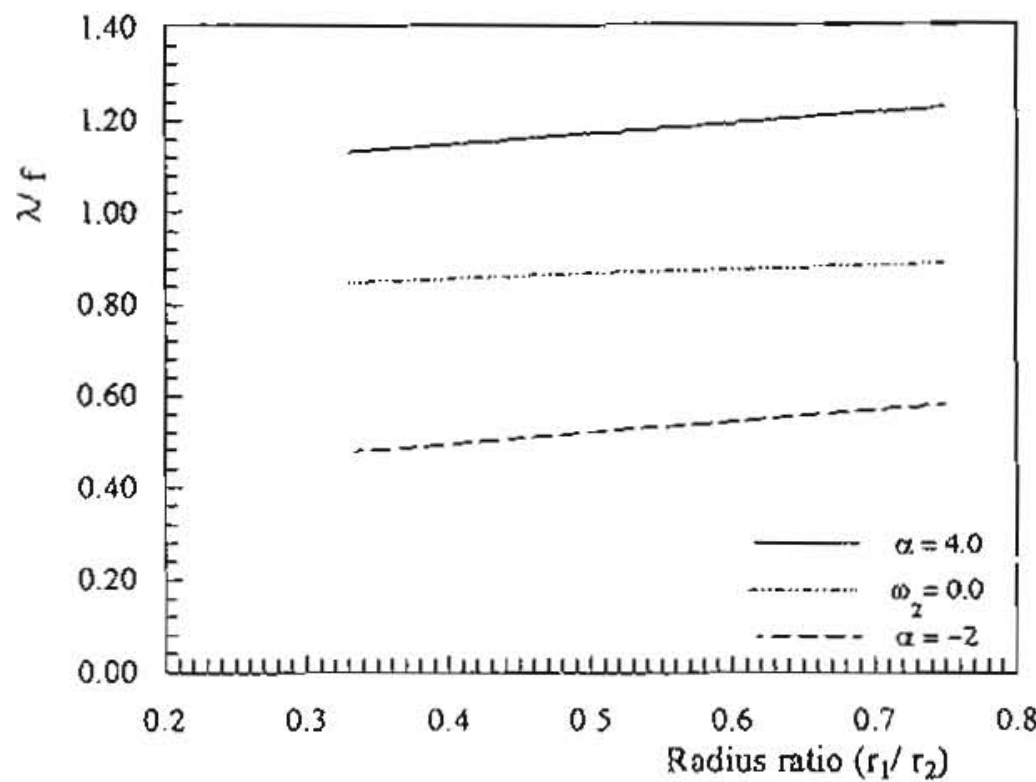

Figure (1-2) Variation of Friction coefficient ratio with radius ratio at different angular velocity ratios for $V_{z_{\text {mem }}}=50(\mathrm{~m} / \mathrm{s})$

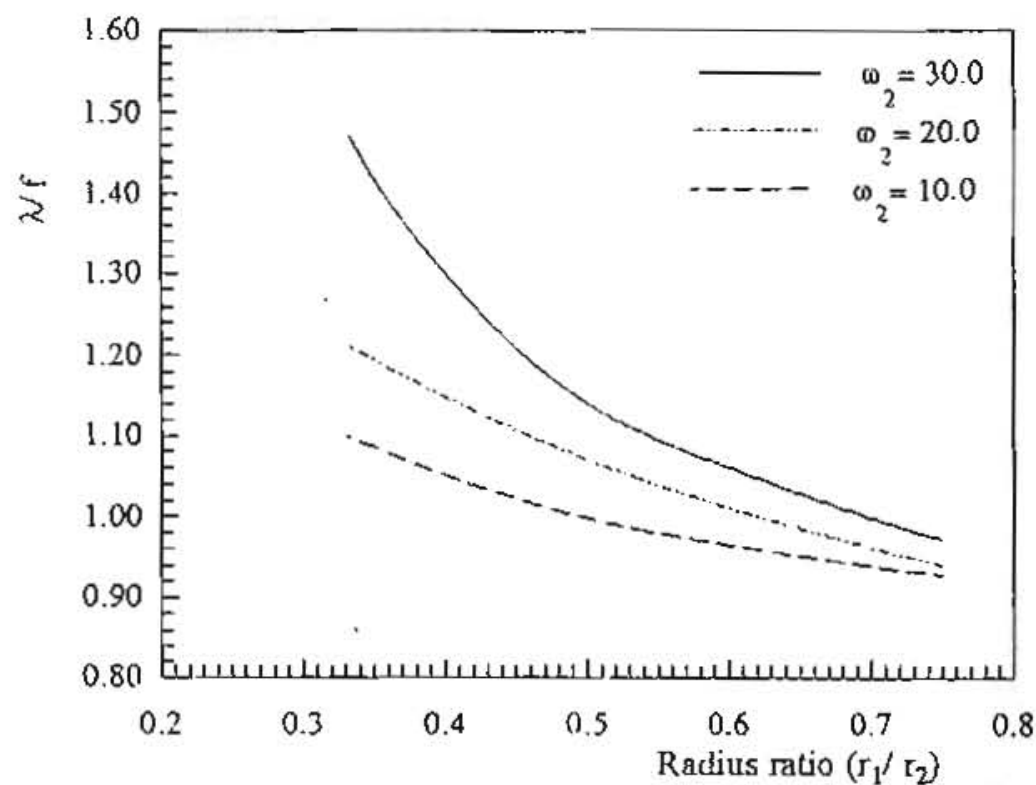

Figure (1-b) Variation of friction coefficient ratio with radius ratio for outer cylinder rotating only $\left(\omega_{1}=0.0\right)$. 


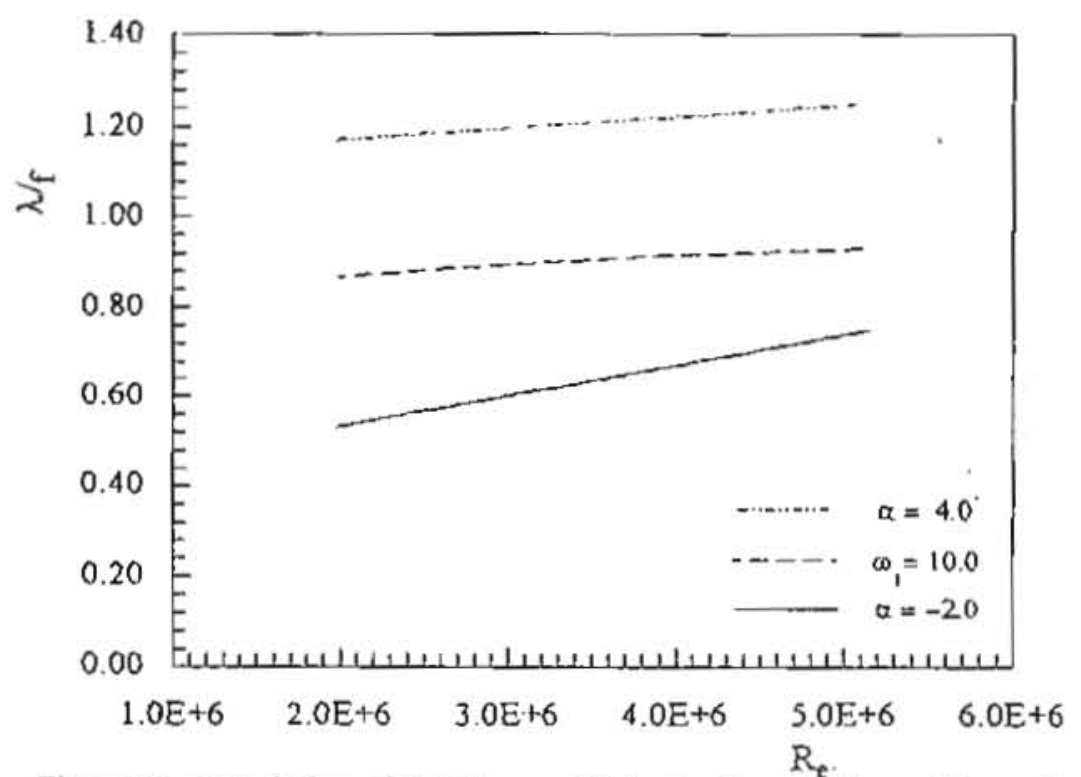

Figure (2-a) Variation of Friction coefficient ratio with Reynolds number for different angular velocity ratios for a gap of radjus ratio $=0.5$

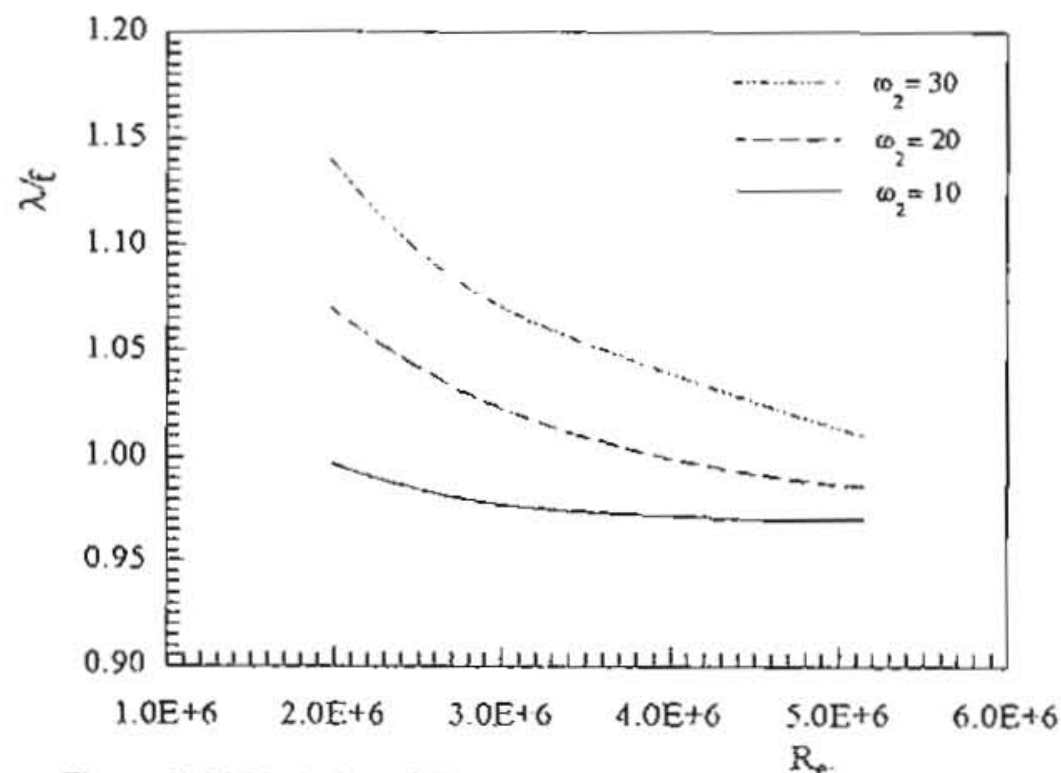

Figure (2-b) Variation of friction coefficient ratio with Reynolds number for outer cylinder sotating only $\omega_{1}=0$. 

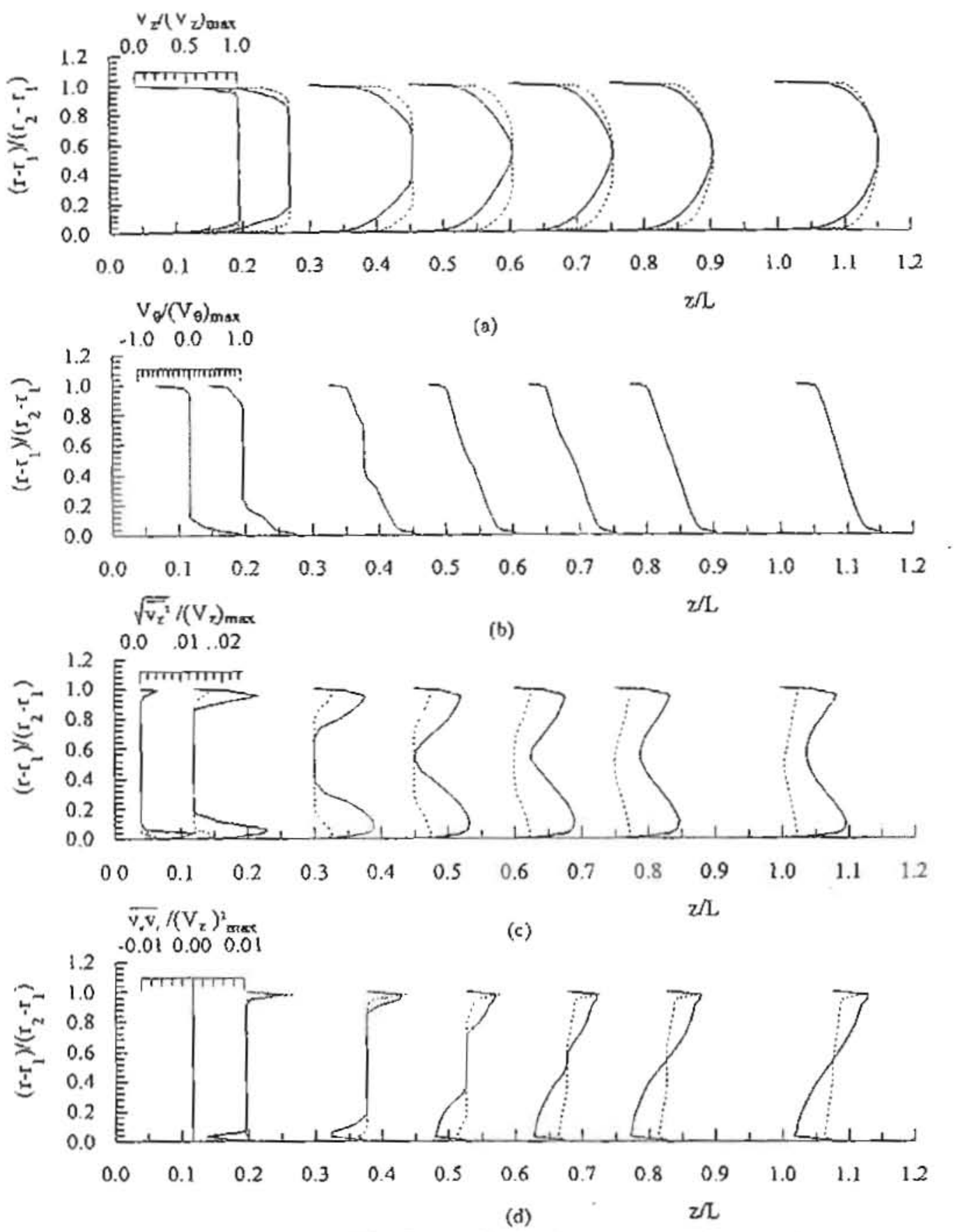

Figure (3) Development of the flow with the downstream distance for radius ratio $=0.75$ with aspect radio $=50.0$ and $R_{c}=6.22 \times 10^{5}$ at $\alpha=-2.0$ Rotating Cylinders. - Stationary cylinders. 

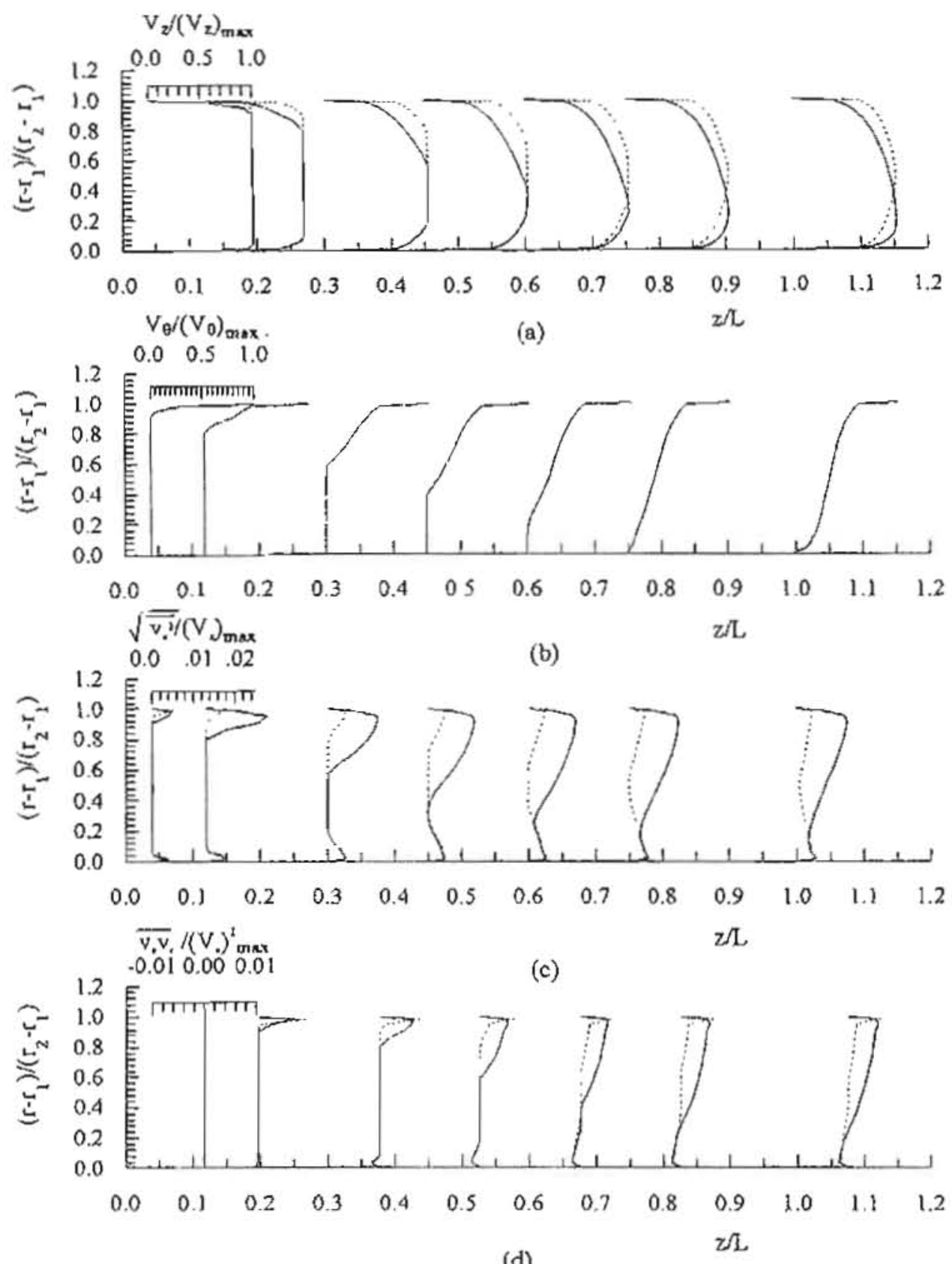

(d)

Figure (4) Development of the flow with the downstream distance for radius ratio $=0.75$ with aspect radio $=50.0$ and $R_{e}=6.62 \times 10^{5}$ at $\omega_{1}=0.0$ Rotating cylinders. Stationary cylinders. 

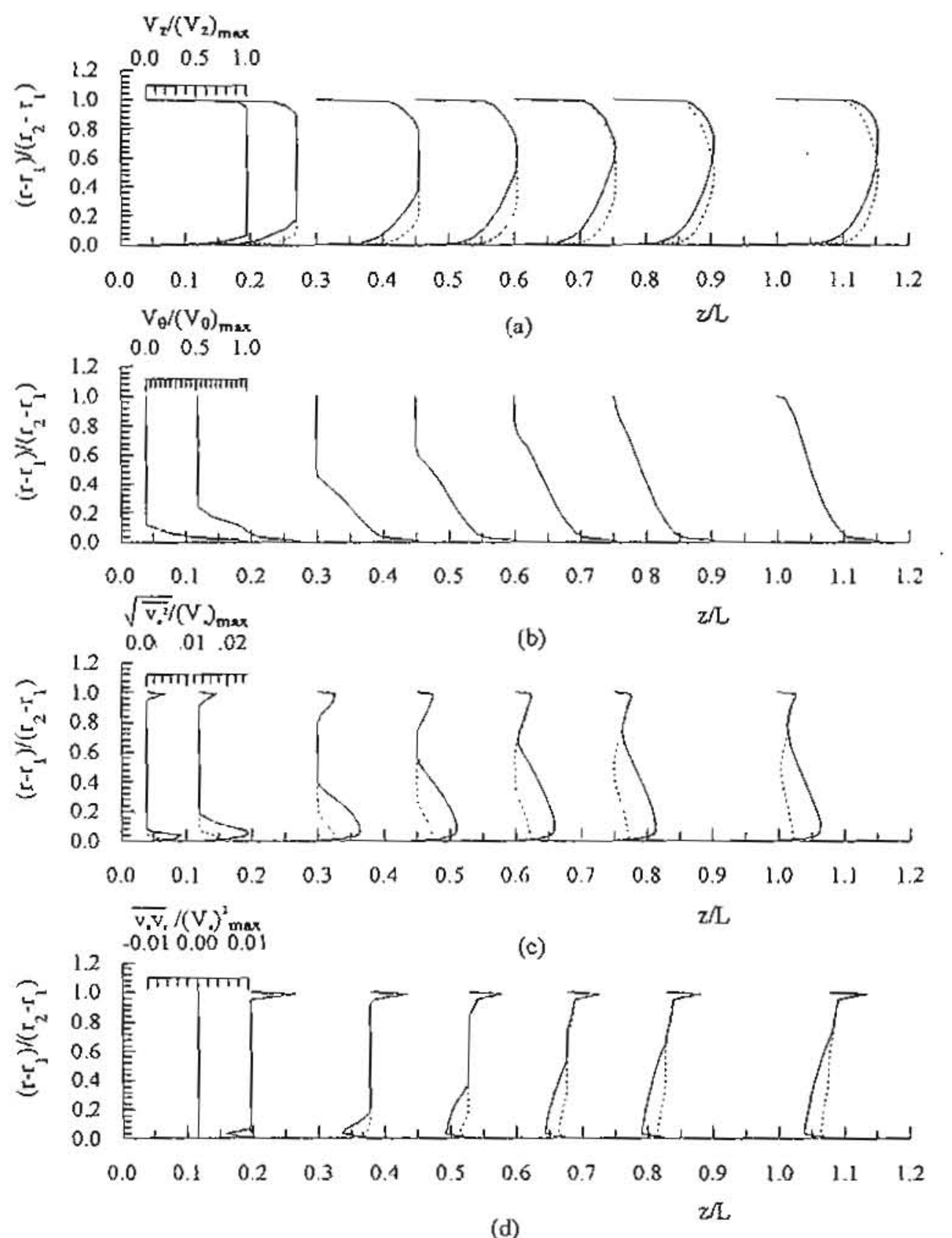

(d)

Figure (5) Development of the flow with the downstream distance for radius ratio $=0.75$ with aspect radio $=50.0$ and $R_{e}=6.62 \times 10^{5}$ at $\omega_{z}=0.0 \& \omega_{1}=10$. - Rolating cylinders. 

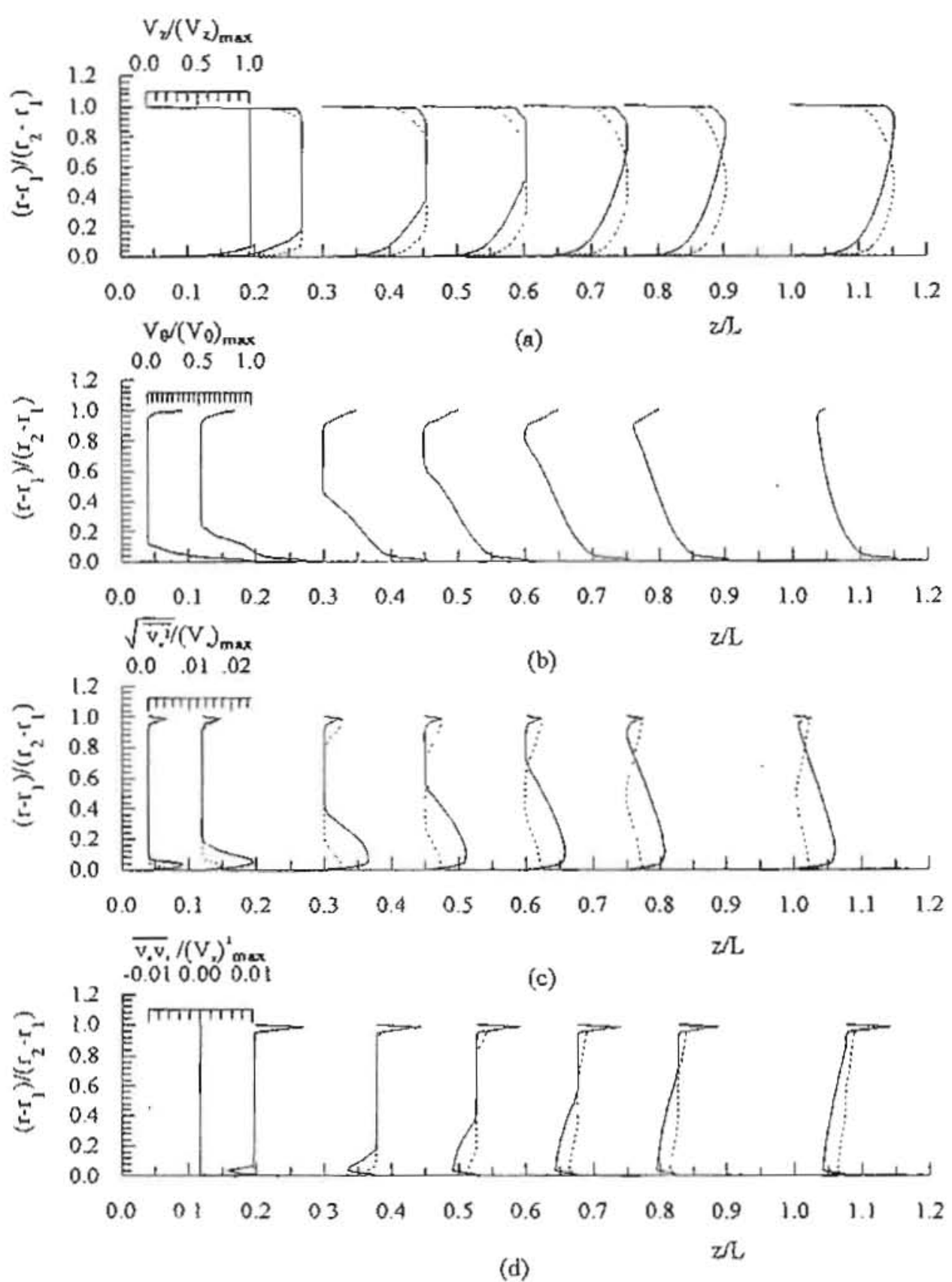

Figure (6) Development of the flow with the downstreara distance for radius ratio $=0.75$ with aspect radio $=50.0$ and $R_{e}=6.62 \times 10^{5}$ at $\alpha=4.0$ Rotating cylinders. Stationary cylinders. 

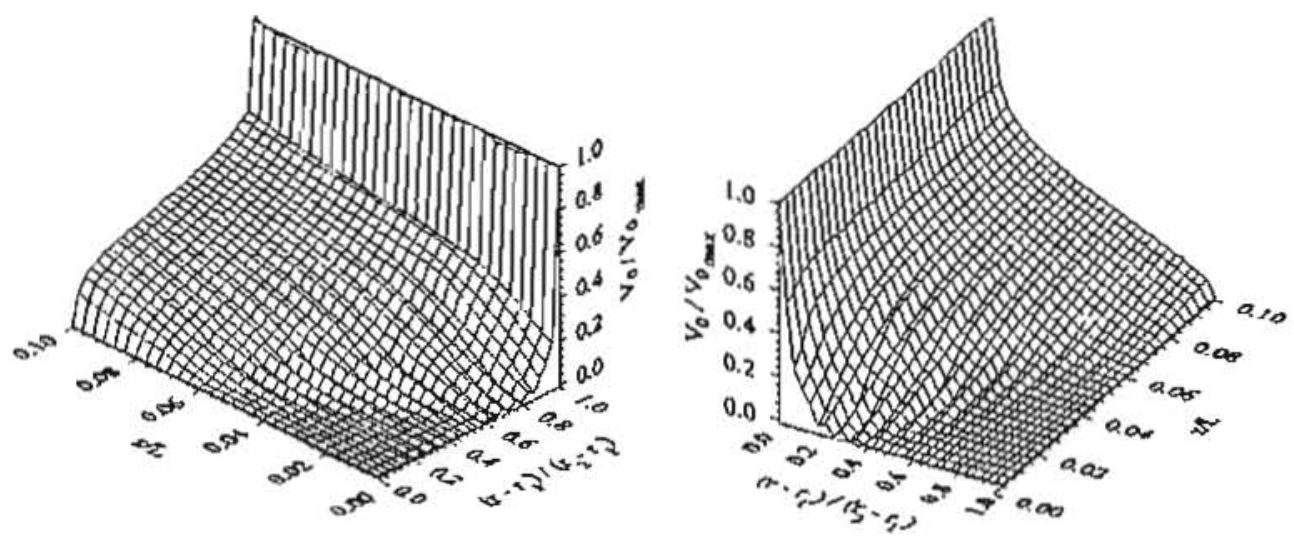

$\omega_{1}=0.0$

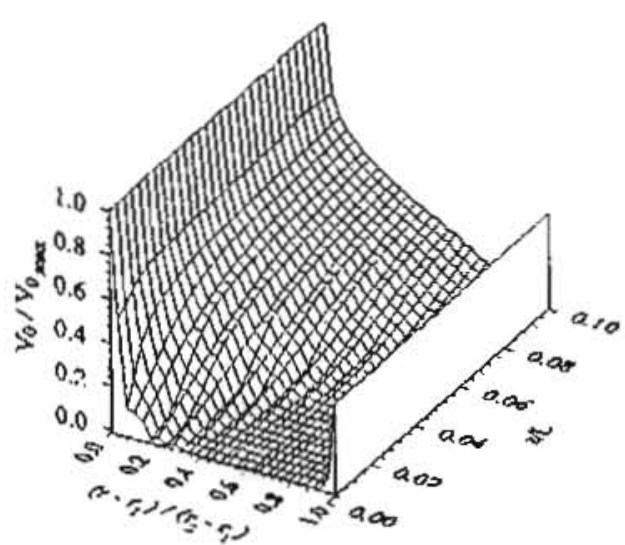

$\alpha=4.0$

$$
\omega_{2}=0.0
$$

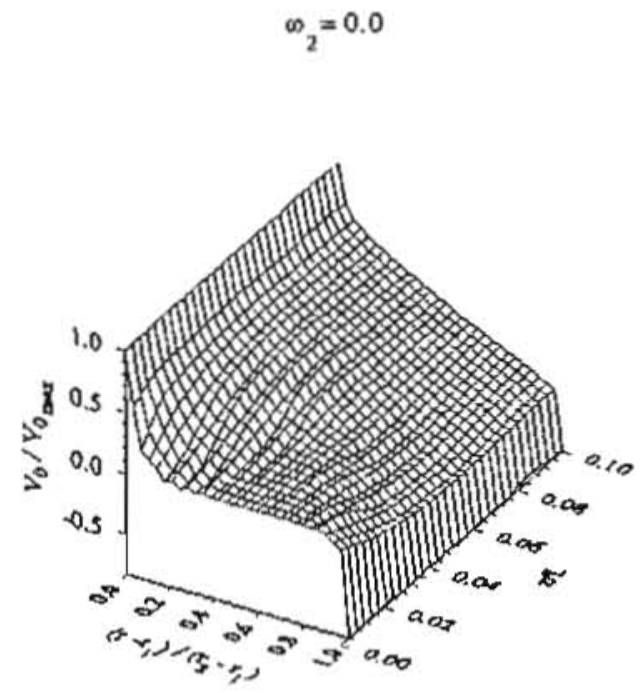

$\alpha=-2.0$

Figure (7) Three-dimensional representation of the predicted tangintial mean velocity for different angular velocity ratios at radius ratio $=0.6$ and $R_{e}=6.62 \times 10^{5}$ 

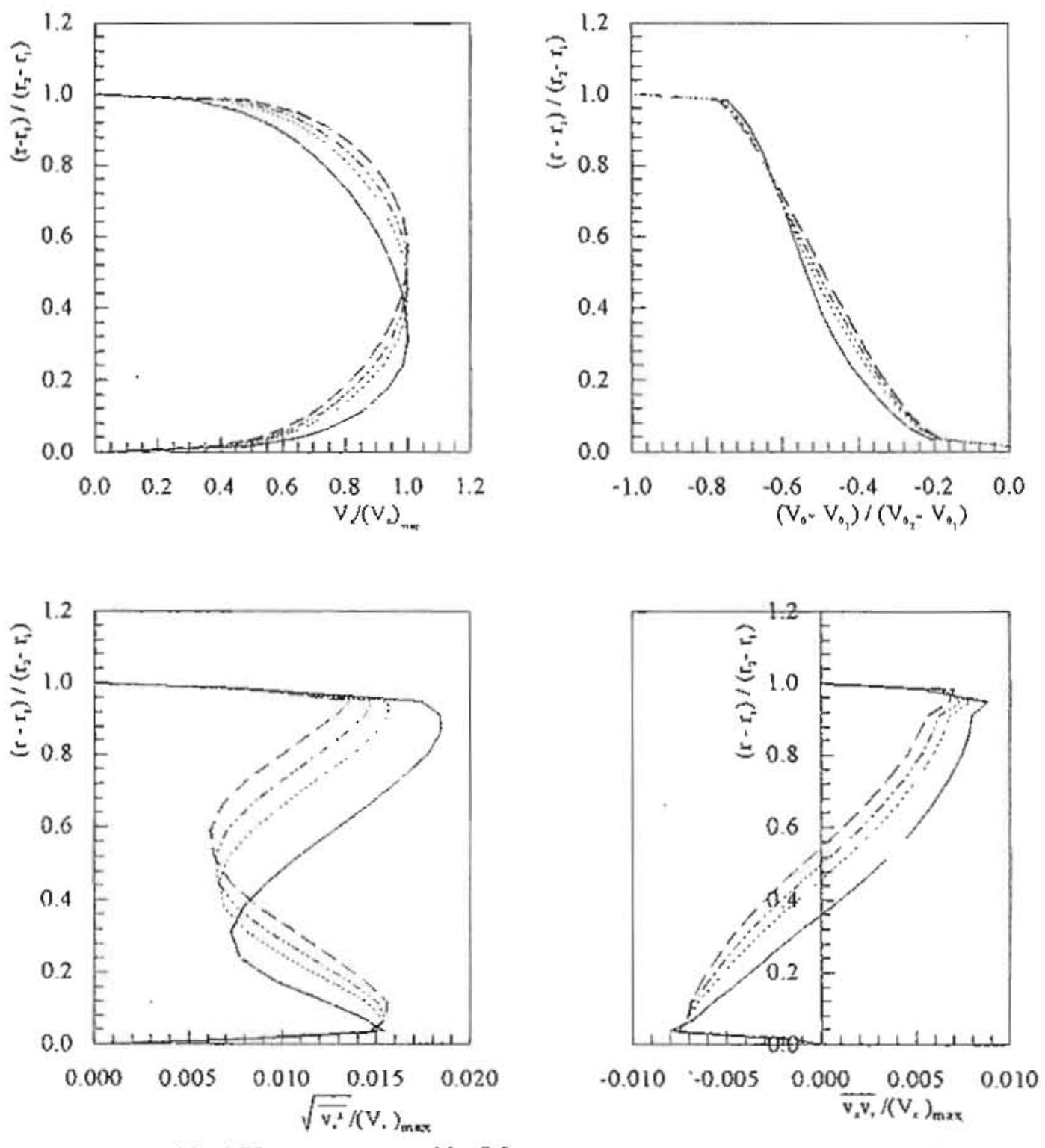

Figure (8) Variation of fully developed flow parameters with radius ratio at aspect ratio $=50 \& \quad \omega_{1} / \omega_{2}=-2.0$. 


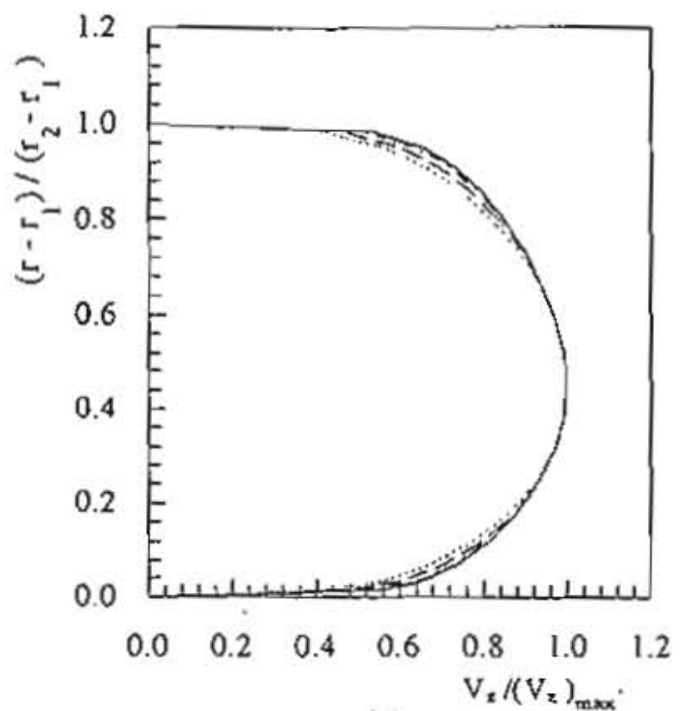

(a)

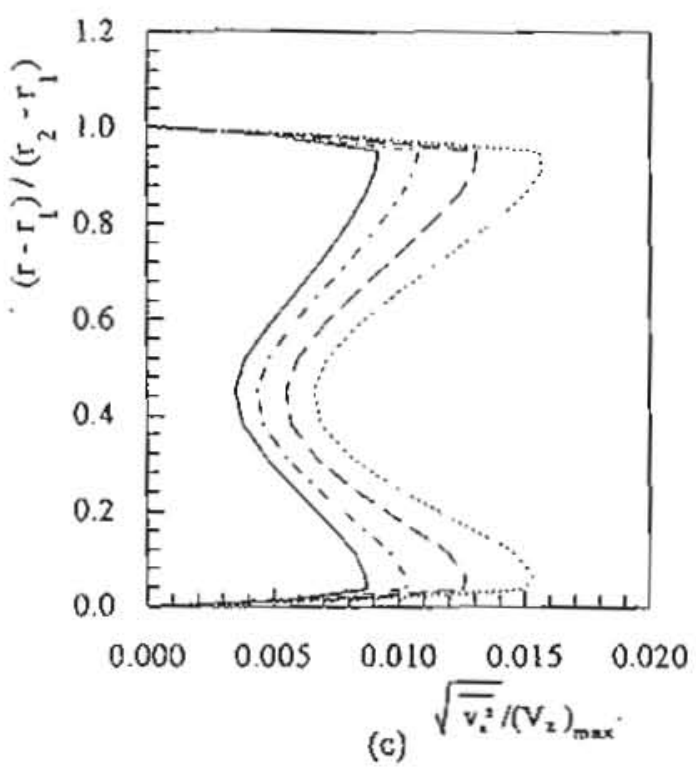

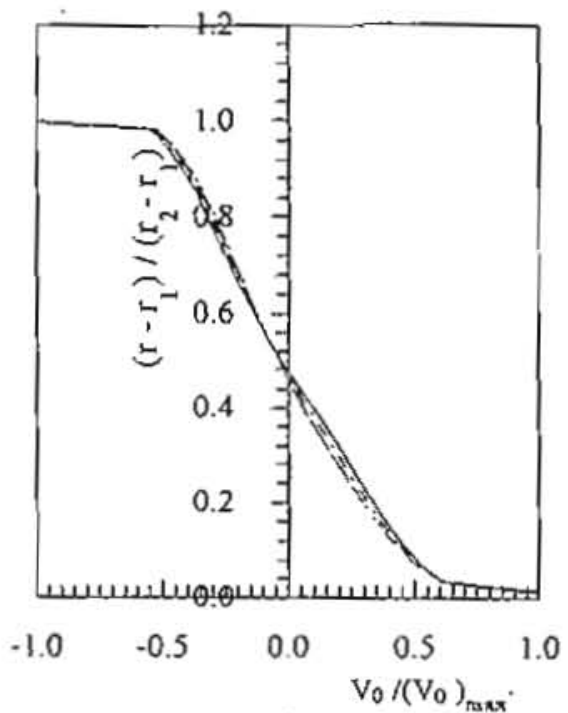

(b)

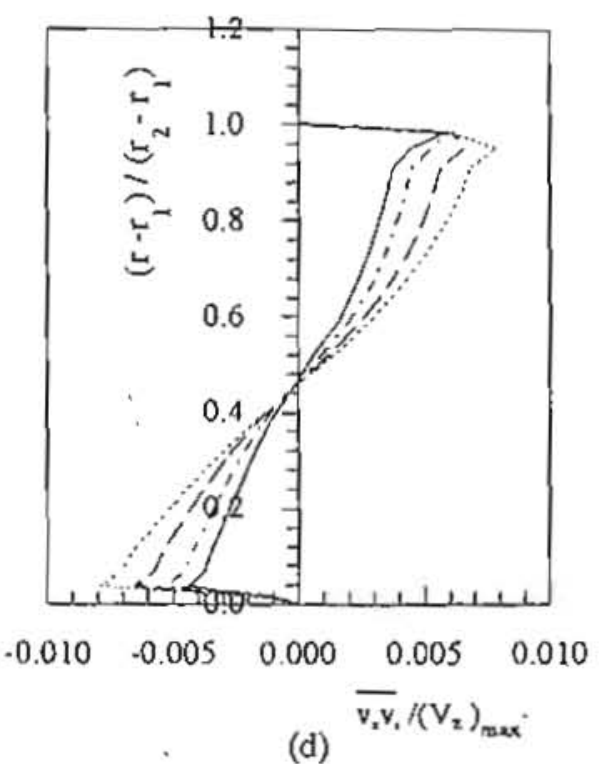

(d) $\overline{v_{2} v_{1} /\left(V_{z}\right)_{\max } \cdot}$

$R_{c}=1.98 \times 10^{6} . \cdots R_{e}=2.78 \times 10^{6} \cdots \cdots \cdot R=3.97 \times 10^{6}$. $R=5.16 \times 10^{6}$

Figure (9) Variation of flow parameters with Reynolds number for fully developed fiow at aspect ratio $=33 \& R_{1} / R_{2}=0.5$ and $\omega_{1} / \omega_{2}=0.5$ :
a) Axial mean velocity.
b) Tanginial mean velocity
c) Turbulence intensity distribution.
d) Shear stress distribution 